\title{
La bibliothèque du traducteur
}

Amyot et ses exemplaires de travail

\section{Romain Menini}

\section{CpenEdition}

\section{Journals}

Édition électronique

URL : http://journals.openedition.org/aes/1483

DOI : 10.4000/aes.1483

ISSN : 2258-093X

Éditeur

Laboratoire LISAA

Référence électronique

Romain Menini, «La bibliothèque du traducteur », Arts et Savoirs [En ligne], 10 | 2018, mis en ligne le 20 novembre 2018, consulté le 19 avril 2019. URL : http://journals.openedition.org/aes/1483 ; DOI :

$10.4000 /$ aes. 1483

Ce document a été généré automatiquement le 19 avril 2019.

Centre de recherche LISAA (Littératures SAvoirs et Arts) 


\title{
La bibliothèque du traducteur
}

\author{
Amyot et ses exemplaires de travail
}

\author{
Romain Menini
}

In memoriam Françoise Frazier

1 Jacques Amyot ${ }^{1}$, évêque d'Auxerre, s'éteint le 6 février 1593. L'inventaire dressé après son décès fait une place importante à sa bibliothèque; ce document nous offre un aperçu remarquable des livres qui, entre autres biens du prélat, entouraient encore, à ses derniers jours, le plus grand traducteur de son siècle. On doit à Sylvie Le Clech-Charton une édition commentée de cet inventaire ${ }^{2}$, dont l'original se trouve aujourd'hui aux Archives départementales de l'Yonne. Le catalogue de livres compte 164 items. Malgré les déformations des titres et des noms d'auteur - la faute au scribe qui copie sous la dictée -, cette liste donne à l'historien une idée assez claire de ce qu'était la bibliothèque de l'évêque: une collection où domine le latin et qui fait la part belle à la culture ecclésiastique (exégèse biblique, apologétique, Pères de l'Église).

Le Sommaire de la vie de Messire Jacques Amyot, que Fédéric Morel le Jeune fit placer en tête de sa réédition des Vies de Plutarque, nous donne une image de l'évêque à l'étude qui concorde parfaitement avec l'inventaire réel de ses livres :

Se voyant parvenu à la qualité d'Evesque, et considerant qu'outre la capacité qu'il avoit aux lettres humaines et en la Philosophie, la Theologie luy estoit necessaire pour s'aquitter condignement de ceste charge si pesante, il se resolut d'y employer plus de temps qu'il n'y avoit encore mis, et partant outre les livres sacrez de la saincte Bible dont il avoit la lecture fort faimilere, il se mit à lire très-serieusement les escrits des SS. Peres tant Grecs que Latins, et entre autres ceux de S. Jean Chrysostome et ceux de S. Augustin fort particulierement et avec une diligence extreme [...]. ${ }^{3}$

3 Sylvie Le Clech-Charton nous apprend en outre que si Amyot avait à sa disposition plusieurs ouvrages de méditation ou de controverse «représentatifs du renouveau spirituel post-conciliaire $»^{4}$, on n'a point trouvé à sa mort de livres hérétiques contrairement à la bibliothèque de Pontus de Tyard, qui comptait du reste quatre fois plus de volumes 5 .

4 La collection du pieux helléniste ressemble en somme, par sa teneur, à bien des bibliothèques ecclésiastiques de son temps - si ce n'est, fait notable, que les ouvrages en 
langue grecque y sont sensiblement plus nombreux. S'y esquisse le portrait d'un évêque de la Contre-Réforme attaché à sa vocation pastorale et s'étant muni des instruments nécessaires à son office auprès des fidèles.

\section{Reconstituer la bibliothèque de l'évêque?}

5 À la lumière de cet inventaire - aux informations parfois lacunaires -, nul n'a tenté, semble-t-il, de retrouver les exemplaires réels qui sont aujourd'hui Dieu sait où, dispersés à coup sûr dans les collections publiques et privées. Notre contribution ne se fixe pas un tel dessein, inattingible en l'état - et vers lequel seule une recherche collective pourra tendre, armée de la patience nécessaire. Mais les trois exemples suivants auront peut-être l'heur de montrer la voie de la reconstitution :

6 - «Les opusculles de Plutarque en grec in-folio, impression de Basle, couvert de veau rouge, prisé cinquante solz, pour ce, L s.» ( $\mathrm{n}^{\circ} 5$ de l'inventaire). Il s'agit selon toute vraisemblance du volume des Moralia (Bâle, Froben et Episcopius, 1542) sur lequel Amyot effectua le travail philologique si remarquable qui préluda à sa traduction complète ; conservé à la Bibliothèque nationale de France (Rés. J. 103 - récemment numérisé), l'exemplaire porte sur son premier plat la mention « Plutarchi opuscula moralia / manu Jacoby Amyot annotata»[fig. 1]. Les marges de cet exemplaire unique recèlent des trésors de variantes qui n'ont cessé de nourrir le travail des éditeurs ultérieurs des Moralia [fig. 2] - et qui constitueront encore, dans l'édition en cours des Euvres morales et meslées, la ressource principale de l'apparat critique conçu pour accompagner le texte français du «sçavant translateur " ${ }^{6}$ ('expression, si juste, est de Du Bellay).

Fig. 1 Plutarque, Moralia

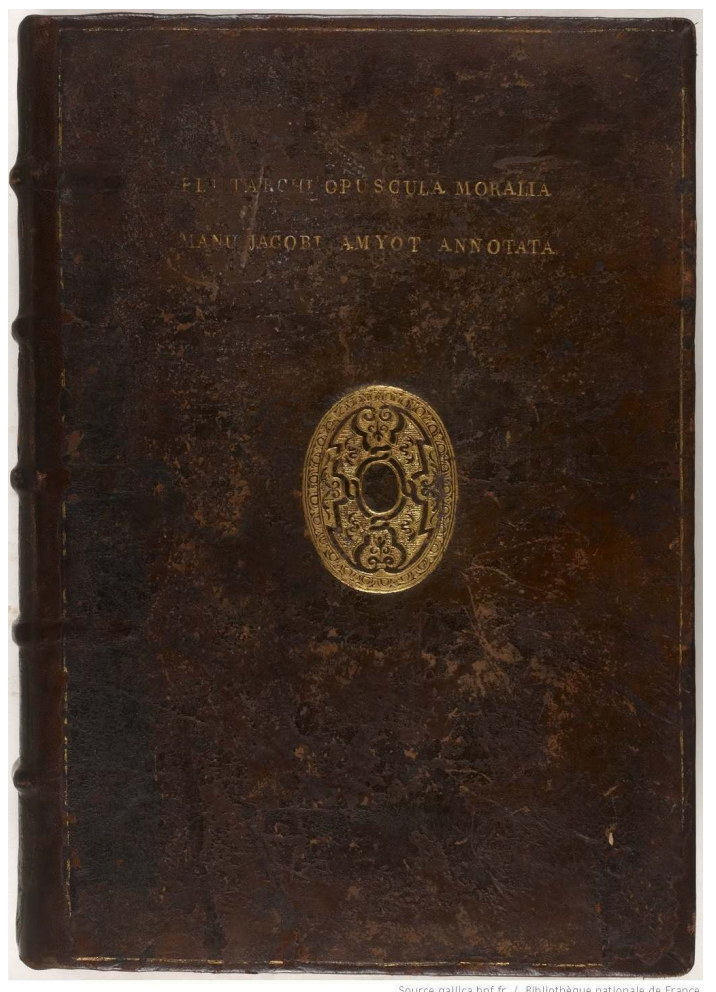

Bâle, Froben et Episcopius, 1542, exemplaire d'Amyot [BnF Rés. J. 103], premier plat avec fer de reliure. Num. Gallica. 
Fig. 2 Plutarque, Moralia

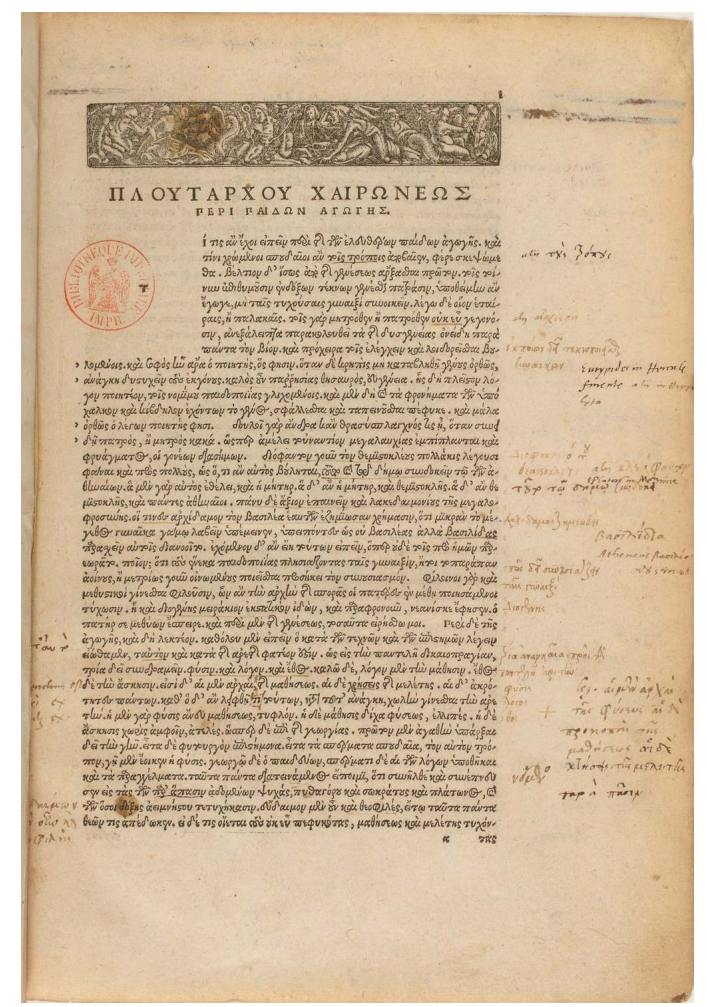

Bâle, Froben et Episcopius, 1542, exemplaire d'Amyot [BnF Rés. J. 103], p. 1, notes autographes. Num. Gallica

7 - «Platina, de vitis pontificum, in-folio, impression de Louvain, couvert de veau noir, prisé vingtz solz» ( $\left.\mathrm{n}^{\circ} 74\right)$. Un exemplaire du De vitis pontificum (Venise, F. Pincio, 1511), porte en page de titre le nom "Amyot "; la base "Provenance » de la Bibliothèque municipale de Lyon, qui le conserve, signale cette mention (non autographe ?) dans son catalogue en ligne. L'exemplaire (Rés. Ms. 195) comporte aussi de nombreuses annotations manuscrites de la main de Benoît Court et de son frère Léonard ${ }^{7}$, possesseurs précédents de l'exemplaire. Bien que la mention de «Louvain » soit discordante dans l'inventaire, la reliure de basane brune a toute chance d'être le « veau noir » évoqué.

8 - «L'histoire d'eclesiastique, de Solo [sic], de Socrates, Theorars [sic pour Theodoret] et aultres, impression de Robert Estienne, in-folio, couverte de veau rouge, prisé demy escu ». Si nos conclusions paléographiques sont exactes, le volume en question est passé en vente récemment à Paris (Drouot, 27 juin 2017) : cet exemplaire de l'édition grecque de l'Histoire ecclésiastique par Eusèbe, Socrate le Scholastique, Théodoret et alii, imprimée avec les fameux « grecs du Roy » (Paris, R. Estienne, 1544) se présentait encore, en effet, sous un « veau estampé d'un fleuron central doré et d'un triple encadrement de filets » 8 . Il s'agit donc d'un veau brun, que le greffier nomme à plusieurs reprises dans l'inventaire « veau rouge » pour le distinguer des reliures plus sombres: ainsi des Moralia précités, encore aujourd'hui en veau brun. Cet exemplaire d'Eusèbe comporte des notes philologiques (emendatio) qui nous paraissent en tous points semblables à celles dont Amyot orna les marges de son Héliodore et de ses Plutarque, tant par leur nature et leur formulation que par leur calibre et leur ductus (voir la page reproduite dans le catalogue de vente [fig. 3]). 
Fig. 3 Eusèbe de Césarée et alii, Histoire ecclésiastique

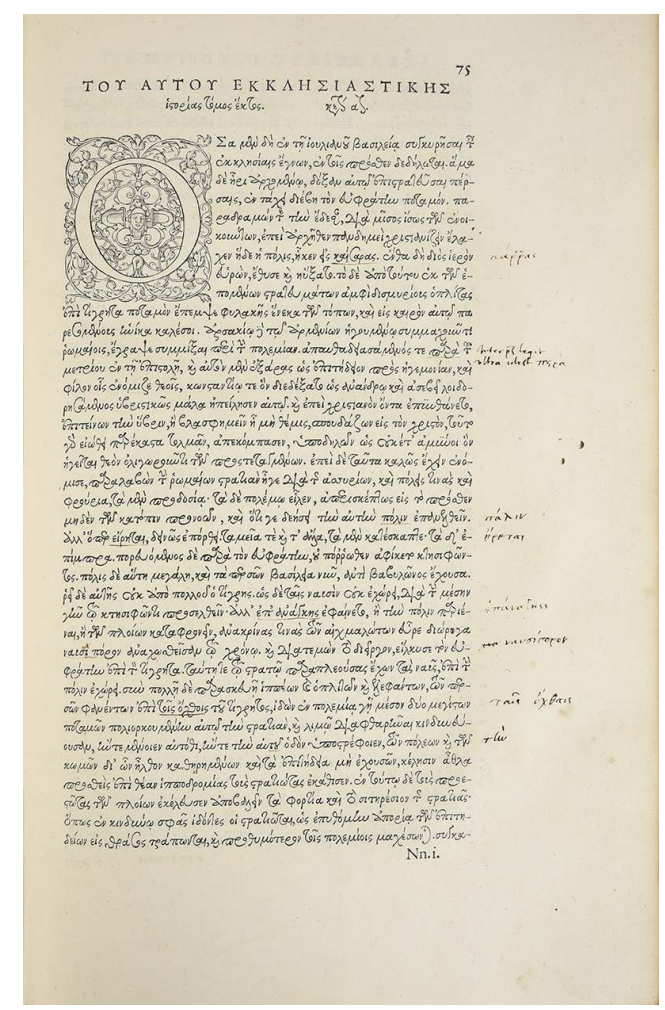

Paris, R. Estienne, 1544 (catalogue Couteau-Bégarie \& Associés. Livres anciens et modernes. 27 juin 2017, lot n 40).

Trois ouvrages sur cent soixante-quatre - c'est fort peu. Encore s'agit-il de voir à quel point la tâche est rude : il semble que Jacques Amyot n'ait que rarement porté son nom ou son ex-libris sur les volumes dont il fit usage ${ }^{9}$. L'une des seules chances d'identifier avec quelque degré de certitude un exemplaire de la bibliothèque de l'évêque pourrait être de reconnaître sa main en marge d'un volume (éventuellement conservé dans sa reliure ancienne qu'avait décrite le greffier). Encore faut-il que lesdits livres portent des marginalia... En la matière, les éditions grecques offrent un terrain de chasse particulièrement intéressant : le philologue était amené à amender les textes imprimés, et il est possible d'identifier avec confiance sa main d'helléniste qui reporte des variantes.

L'inventaire signale aussi des « opusculles de Plutarque en francoys, traduictes [sic] par le deffunct, in-folio, impression De Vostre [sic pro De Vascosan]» $\left(n^{\circ} 4\right)$. Nous ignorons aujourd'hui où peut bien se trouver ce volume, mais il est fort probable qu'il s'agisse de l'exemplaire (Paris, M. Vascosan, 1572) qui contenait les dernières corrections manuscrites de l'évêque en ses CEuvres morales et meslées - ces ultimes repeints qui furent eux-mêmes copiés, vraisemblablement par son secrétaire Regnaud Martin, dans un manuscrit conservé aujourd'hui à la Bibliothèque Ambrosienne de Milan ${ }^{10}$.

\section{Et la bibliothèque du traducteur?}

11 Sans retirer à l'inventaire son importance historique, il faut reconnaître que, pour celui qui s'attache à ce «traducteur français » dont Antoine Berman a souligné le statut et la stature exceptionnels ${ }^{11}$, le catalogue post mortem déçoit un peu. Sylvie Le Clech-Charton 
souligne «le caractère incomplet du fonds » et s'étonne à bon droit de "l'absence de livres de littérature ", avant de s'interroger : "s'il [sc. Amyot] a fait don à l'un de ses neveux ou à son frère Jean, qui étaient laïcs, ces ouvrages ne figurent pas dans l'inventaire $»^{12}$. Nous ignorons comment la bibliothèque du traducteur - celle qui permit à l'helléniste de fréquenter aussi (et avec quelle expertise!) l'Antiquité païenne - vint à quitter le cabinet de travail d'Amyot, en 1593 ou plus tôt : par son secrétaire Regnaud Martin, qui eut accès aux traductions françaises annotées? par Jean Amyot, frère de l'évêque, qui mourut un an après Jacques ${ }^{13}$ ? par l'un des neveux du traducteur: Jean Desbourneaux - à qui Amyot avait fait don, en 1589, de tous ceux de ses livres qui se trouvaient à l'hôpital des Quinze-Vingts -, Louis Desbourneaux ou Nicolas Amyot qui, avec le précédent, assista à l'inventaire après décès ${ }^{14}$ ?

Signalons d'ores et déjà, dans l'inventaire, l'absence de deux volumes grecs à l'importance remarquable : - l'exemplaire in-quarto de l'édition princeps des Éthiopiques d'Héliodore (Bâle, Herwagen, 1534) à partir duquel Amyot put réaliser sa traduction à succès parue en 1548 (puis une version révisée "sur un ancien exemplaire escript à la main, par le translateur" en 1559). La Bibliothèque Sainte-Geneviève conserve aujourd'hui ce précieux exemplaire ${ }^{15}$, abondamment annoté par l'helléniste, qui - une fois tournée la page de titre où se trouvent mentionnés la Bibliothèque Vaticane et l'historien byzantin Georgius Cedrenus ${ }^{16}$ - avait reporté en marge presque uniquement des variantes sèches, pour ainsi dire, sans glose ni renvoi à d'autres loci similes ${ }^{17}$.

14 - l'édition aldine des Vies de Plutarque (Venise, 1519) dont Amyot a rempli les marges pour établir le texte grec à partir duquel il effectua sa traduction parue en 1559 (puis révisée en 1565 et 1567). L'exemplaire est aujourd'hui consultable à la Bibliothèque de l'Arsenal sous la cote Ms. 8411. Les plats de la reliure sont ornés du même fer ovale que sur l'exemplaire des Moralia de Tolbiac $^{18}$, et un feuillet de garde porte une inscription identique: "Plutarque de messire Jacques Amyot, vivant evesque d'Auxerre et grand Aumosnier de France. Rapporté de Melun, le samedy xiiii jour de novembre 1609, par mon nepveu $M^{\text {re }}$ Pierre Regnault, procureur du Roy au siege presidial dud. Melun.» [fig. 4].

Fig. 4 Plutarque, Moralia

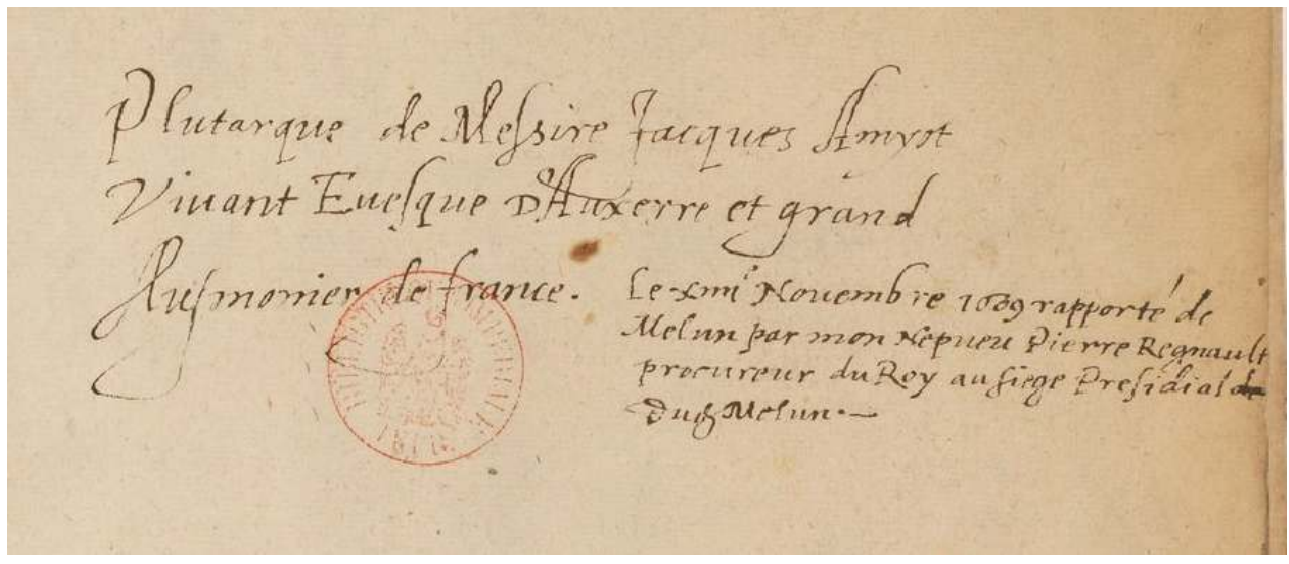

Bâle, Froben et Episcopius, 1542, exemplaire d'Amyot [BnF Rés. J. 103], feuillet de garde. Num. Gallica. 
excepte les Moralia de Plutarque - l'absence à peu près totale des livres de l'Antiquité païenne, tout autant que de leurs commentaires humanistes (une exception: les Hieroglyphica de Valeriano, $\mathrm{n}^{\circ} 16$ ). Or cette ressource culturelle et philologique fut le recours privilégié du traducteur de Plutarque. À qui voudrait reconstituer, ne serait-ce que virtuellement, la bibliothèque de l'helléniste, il faut donc trouver d'autres voies, l'inventaire des biens du prélat ne pouvant suffire. En l'état de nos connaissances, l'une des manières les plus fiables de comprendre comment Amyot travaillait, et quels outils il possédait à sa table de travail (ou sur sa roue à livres), est certainement de sonder les marges de ses livres annotés. Nous proposons ici d'interroger, par-dessus les épaules du « sçavant translateur ", les marges de ses exemplaires de travail - en particulier celui des Moralia déjà mentionné.

\section{Bibliotheca in margine}

Robert Aulotte, dans son étude de référence, a donné de précieuses indications sur la bibliothèque réelle et probable du traducteur - et plus généralement sur ce que nous appellerions aujourd'hui ses instruments de travail, dans toute leur diversité2 ${ }^{2}$. Outre l'exemplaire de la Bibliothèque nationale de France et les traductions disponibles de son temps, c'est notamment sur les manuscrits consultés par Amyot que les recherches du critique ont porté.

On n'ignore point le soin avec lequel, très tôt, Amyot l'helléniste mena sa recherche des meilleures variantes, en particulier dans les manuscrits français et italiens qu'il put avoir à sa disposition. En 1554, l'avertissement « Aux lecteurs » qui précède sa traduction des Sept livres des histoires de Diodore de Sicile mentionne l'importance du manuscrit « de la 
librairie de saint Marc à Venize » dont il s'est servi ${ }^{23}$. Cinq ans plus tard, sa traduction des Amours pastorales de Daphnis et Chloé (Paris, pour V. Sertenas, 1559) devance de trente-neuf ans l'editio princeps du texte de Longus (Florence, F. Giunta, 1598); on sait désormais qu'Amyot put donner cette version pionnière grâce au Parisinus 2895, un manuscrit que Girolamo Fondulo avait fait venir à Paris ${ }^{24}$, en 1539 , à l'intention du roi. Nous avons déjà évoqué la parution, cette même année 1559, la révision de la traduction des Éthiopiques d'Héliodore à partir d'un manuscrit de la Vaticane difficilement identifiable.

En s'attachant aux Moralia comme avant lui Sturel s'était dévoué à l'étude des Vies, Aulotte listait dix-huit manuscrits, essentiellement parisiens et italiens, auxquels Amyot pouvait recourir, au moins ponctuellement. Mais évaluer l'utilisation exacte et précise que fit le traducteur de chacun de ces manuscrits reste une tâche mal aisée. Marcel Cuvigny, après avoir identifié le Parisinus 2074 derrière une annotation d'Amyot en marge de son exemplaire (p.703: «in vetere manuscripto bibliothece Reginoe legitur etc.») ajoutant ainsi un manuscrit de plus à la glane d'Aulotte -, nous mettait en garde : « S'il a, comme il est probable, collationné les manuscrits du roi et de la reine-mère, il faut bien convenir que mainte leçon excellente lui a échappé. $»^{25}$

Ainsi ne faudrait-il pas imaginer Amyot procédant à quelque collation exhaustive dans chacun des codices mis à sa disposition, fût-ce dans la bibliothèque royale. Ses relevés ne pouvaient être systématiques - et la difficulté à laquelle ont été confrontés les codicologues désireux de lire dans les marges de l'Héliodore grec une source unique peut servir de leçon de méthode. Du reste, une telle précaution n'entre pas en contradiction avec les recherches d'Aulotte, qui ont eu le grand mérite d'attirer aussi l'attention sur l'importance, pour l'helléniste au travail, des recueils contemporains de variantes manuscrites ainsi que des traductions latines disponibles; autant de ressources capables de concurrencer les manuscrits, et pour tout dire de s'y substituer parfois, parce qu'elles firent gagner au traducteur un temps considérable - en même temps qu'elles rendaient toute future enquête limitée aux seuls manuscrits à peu près impossible.

Il en va de même des volumes imprimés que manipulait Amyot: la circulation des exemplaires, particulièrement celle des in-folio grecs (ayant souvent recueilli, en marge, le travail des érudits européens), nous interdit d'imaginer que le traducteur fut le possesseur de tous les volumes qui lui permirent d'accomplir son grand œuvre. Un exemple nous en convaincra: la critique s'accorde à penser qu'Amyot put user de l'exemplaire aldin des Moralia annoté par Adrien Turnèbe ${ }^{26}$ (désormais BnF Rés. J. 94), dans lequel on trouve plusieurs leçons dont il put profiter, issues elles-mêmes parfois de collections plus anciennes (Lascaris, Leonico Tomeo, Scipion Carteromachos, Gianotti). En théorie, il faudrait donc veiller à distinguer la collection personnelle de la bibliothèque de travail, afin d'être le plus fidèle possible à la nature des sociabilités érudites de la Renaissance, au sein desquelles la circulation des exemplaires était capitale - la formule "et amicorum » et ses équivalents, qu'on lit sur tant de livres du XvI ${ }^{e}$ siècle, recouvrant une réalité sociologique de première importance ${ }^{27}$.

Ainsi faut-il imaginer Amyot, au fil des ans, inscrivant sur son exemplaire ces bribes philologiques issues de sources diverses (l'édition aldine, un recueil de variantes, tel manuscrit, une traduction latine, certain locus similis chez les Anciens, etc.), et faisant précéder, parfois, telle ou telle variante d'ordinaire sèche des humbles ornements si caractéristiques du style sténographique de ses marginalia: puto legendum, forte, male, codices legunt, ex veterum codicum fide, aldinus codex, interpres latinus, videntur interpretes legisse, etc. De fait, la philologie est le plus souvent cette discipline de l'ombre qui, jalouse, 
interdit qu'on rende hommage à ses héros discrets : ainsi est-il rare qu'une leçon, même établie ope ingenii, soit rendue à son "inventeur » - qui n'ignorait en rien l'anonymat auquel son travail était voué.

\section{Budæus, Turnebus et les autres}

Les Moralia d'Amyot contiennent malgré tout dans leurs marges certains noms d'érudits qui nous permettent de mesurer à quel point son «Plutarque françois » a profité des travaux des hellénistes contemporains (ou de la génération précédente).

Budous. L'exemplaire aldin des Moralia qu'avait annoté Guillaume Budé n'a pas encore été identifié. Nous savons cependant que, pressé de découvrir le travail d'Érasme, d'Aléandre et des philologues italiens de Venise, il en reçut le contenu en deux temps, immédiatement après que les cahiers furent imprimés ${ }^{28}$. Si le recours à cet exemplaire ne doit pas être exclu, il est cependant vraisemblable que la plupart des mentions de Budé renvoient aux Commentarii linguce grcecee (1529, plusieurs rééd.) - ouvrage majeur qui vint très vite nourrir les dictionnaires grecs publiés dans toute l'Europe humaniste. Amyot avait aussi connaissance des traductions de certains opuscules dues au grand érudit français.

Turnebus (Turnebus). Le cas de l'exemplaire aldin d'Adrien Turnèbe vient d'être évoqué. Amyot connaissait à coup sûr ses Adversaria, mais ils ne lui furent d'aucune utilité pour les Moralia. Sturel a montré que c'étaient surtout aux traductions plutarquéennes de Turnèbe (Septem sapientium convivium, De Syllaba Ei, De oraculorum defectu, De fato, De primo frigido, De animce procreatione) - plutôt qu'à son exemplaire à variantes - que renvoyaient les mentions "Turnebus» dans l'exemplaire d'Amyot ${ }^{29}$. Ainsi, en marge du De defectu, l'annotateur nous renseigne-t-il (p. 245) : "Tornebus ex Timceo Platonis locum hunc putat emendandum "; une confrontation du passage non avec la traduction, mais avec les annotationes de Turnèbe jointes à sa version latine du De defectu confirme que c'est à ces lectiones qu'a recouru Amyot $^{30}$. L'inventaire nous apprend $\left(n^{0} 63\right)$ que l'édition de Philon procurée par Turnèbe (Paris, 1552, in-folio) faisait partie de la bibliothèque de l'évêque, qui avait toute raison de mesurer à sa juste valeur le travail philologique de l'autre grand helléniste de son temps. À ce propos, La Popelinière ne craignait nullement d'attribuer tous les mérites de la traduction des Vies par Amyot... à Turnèbe lui-même (qui mourut en 1565 - trop tôt pour qu'on lui attribuât aussi les Moralia !) :

Les Vies de Plutarque, que Me Jacques Amiot, evesque d'Auxerre, grand aumonier et precepteur de Messeigneurs les enfans de France, mit en françois, à l'aide toutesfois et suivant l'advis de plusieurs gens doctes, de Turnebus entre autres, mon premier precepteur en langue grecque, desquels il tiroit les passages tous tournés [...]. ${ }^{31}$

Par-delà l'exagération de l'historien, tout à la grande gloire de son maître, le témoignage nous éclaire sur le travail collégial qui préluda aux chefs-d'œuvre signés Amyot - les hellénistes faisant souvent aussi, comme on l'imagine, bibliothèque commune.

Aulotte, après Jäger et Sturel, avait recensé les noms des quelques autres érudits cités par Amyot en marge de ses Moralia : Guillaume Philandrier, Théodore (Dirk) Canter, Girolamo Mercuriale, Juste-Lipse - tous auteurs de recueils de varice lectiones compulsés par le savant translateur ${ }^{32}$. L'ouvrage de Dirk Canter, publié seulement en 1574 , ne fut utilisé par Amyot qu'après la première édition des Cuvres morales et meslées (1572). En marge du De superstitione (p.124), on suit par exemple l'helléniste français restituer, grâce à Canter, tels vers d'Archiloque cités par Héraclide du Pont ${ }^{33}$. De plus, Aulotte a donné des 
arguments probants montrant qu'Amyot, bien qu'il ne l'ait jamais mentionné, avait aussi utilisé les Novce lectiones (1566) de Guillaume (Wilhelm) Canter, frère aîné de Théodore. Cela n'a rien d'étonnant, lorsqu'on pense que Guillaume Canter, élève de Dorat, fut l'éditeur d'Euripide en 1571 et que Théodore était appelé à continuer l'œuvre de son frère aîné en recueillant, pour la première fois, les fragments du Tragique ${ }^{34}$. Amyot, comme on sait, avait fait parler Euripide en français quelques années plus tôt ${ }^{35}$; l'évêque d'Auxerre suivait encore avec intérêt les travaux d'une telle fratrie d'hellénistes. Une variante n'a pas de frontière: les marges des Moralia annotés nous montrent la respublica litteraria européenne à l'œuvre.

\section{Auctores classici : quelques éditions identifiables}

Quid des Anciens? La regrettée François Frazier, préparant l'index des auteurs cités par Amyot dans les marges de ses exemplaires, évoquait « une cinquantaine d'entrées, qui vont chronologiquement d'Homère à "Suidas" [i. e. la Souda, dictionnaire byzantin] et comprennent des auteurs grecs et latins de tous les genres, poètes et prosateurs, majoritairement historiens, philosophes, savants et encyclopédistes ${ }^{36}$. La liste des auteurs cités par Amyot est édifiante :

Poètes grecs: Anacréon, Archiloque, Aristophane, Callimaque, Eschyle, Euclide, Euripide, Hésiode, Homère, Ménandre, Pindare, Sappho, Simonide, Sophocle.

Poètes latins : Horace, Juvénal, Ovide, Properce, Stace, Virgile.

Prosateurs grecs: Archimède, Athénée, Denys d'Halicarnasse, Diodore de Sicile, Diogène Laërce, Dion Cassius, Dioscoride, Eusèbe de Césarée, Flavius Josèphe, Hérodote, Hippocrate, Lucien, Pausanias, Plutarque (Vies), Stobée, Strabon, Théophraste, Thucydide, Xénophon.

Prosateurs latins: Aulu-Gelle, Cicéron, Festus, Florus, Lactance, Macrobe, Pline l'Ancien, Sénèque, Suétone, Tacite, Tite-Live.

Soit une grande partie de la littérature ancienne, dans laquelle les historiens, naturellement, se trouvent abondamment représentés. On l'a dit: presque aucun de ces auteurs - païens pour la plupart - ne figure au paradis de l'inventaire post mortem.

Il n'est pas nécessaire de rappeler l'importance de lire les auteurs anciens dans les éditions que les humanistes avaient à disposition. Malheureusement, les renvois aux écrivains classiques que proposent les marges des livres d'Amyot manquent le plus souvent d'un numéro de page nous permettant de remonter à une édition précise. Au moins trois exceptions sont cependant notables.

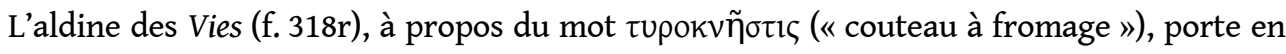
marge : « vide Pollucem libr. 10 p. 24 columna 388 et 389 ». Une vérification du lieu cité montre que c'est à l'édition aldine (1502) de l'Onomasticon que s'est référé Amyot. Le lexique ancien de Julius Pollux s'ajoute donc aux deux autres dictionnaires classiques cités eux aussi dans les marges des Vies comme des Moralia: la somme d'Hésychius et la Souda ("Suidas ») - deux outils de travail nécessaires dont la teneur, à l'époque d'Amyot, avait été intégrée dans les dictionnaires humanistes, qui pouvaient donc aussi servir d'intermédiaire à l'helléniste. En la matière, l'inventaire nous vient en aide : y sont recensés non pas un, mais deux exemplaires de "Lexicon graecolatinum ». S'il est difficile d'identifier l'édition in-folio ( $\left.n^{\circ} 13\right)$, il y a fort à parier que l'exemplaire in-quarto $\left(n^{\circ} 2\right)$, «impression de Lion ", soit le dictionnaire imprimé par Jean Crespin, à Genève, pour Guillaume Rouillé, libraire lyonnais (1566) - soit une refonte portative du volumineux Lexicon publié quatre ans plus tôt par Crespin ${ }^{37}$. Ajoutons enfin que, dans les Moralia, 
Amyot fait deux références (p. 564 et 577) à « Stephanus » : il ne s'agit pas là d'une mention de l'illustre famille des Estienne, mais d'Étienne (ou Stéphane) de Byzance, écrivain byzantin $\mathrm{du} \mathrm{VI}^{\mathrm{e}}$ siècle, auteur d'un important lexique géographique dont les humanistes faisaient leur miel. Amyot trouvait notamment dans ce «De urbibus», titre qu'on lui

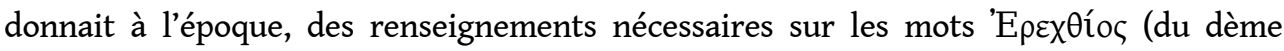

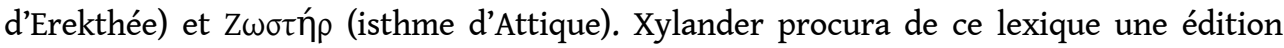
corrigée en 1568, mais Amyot disposait certainement d'un exemplaire plus ancien (aldine de 1502 ou juntine de 1521). Force est de constater, à l'issue de cette enquête rapide, que le traducteur disposait des tout meilleurs outils lexicographiques de sa génération.

L'édition bâloise des Moralia nous mène quant à elle vers deux in-folio importants. Il s'agit d'abord des Deipnosophistes d'Athénée, auxquels fait écho un passage du De pueris mentionnant le poète Sotadès : la note marginale d'Amyot (p. 8) renvoie au folio 307 d'une édition imprimée, qui n'est autre que la deuxième édition de la compilation, publiée en 1535, à Bâle, par J. Walder. Plus maniable que l'aldine de 1514, cette édition d'Athénée qui ne fut traduit en latin qu'en 1556 - comporte des manchettes et des titres courants : le nom du poète Sotadès se lit justement au folio cité par Amyot. [fig. 5]

Fig. 5 Plutarque, Moralia

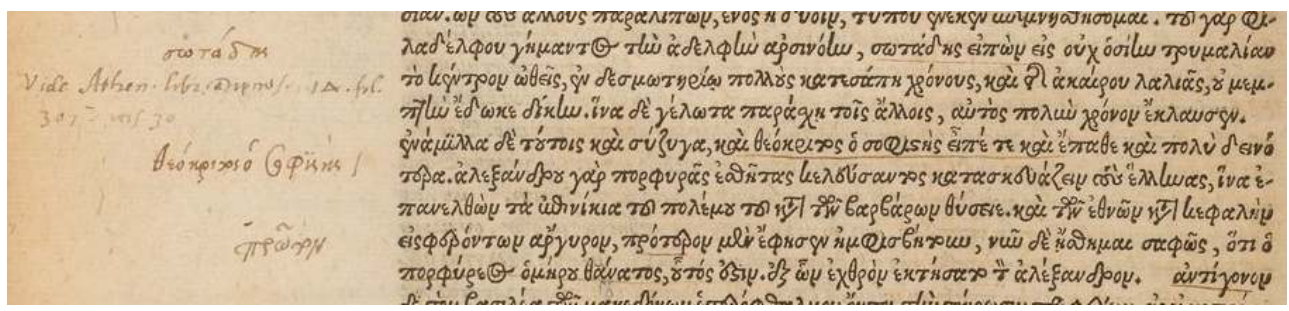

Bâle, Froben et Episcopius, 1542, exemplaire d'Amyot [BnF Rés. J. 103], p. 8 (renvoi autographe à Athénée, Deipnosophistes, Bâle, J. Walder, 1535, f. 307r). Num. Gallica.

Le traducteur a par ailleurs recouru, à plusieurs reprises, comme le prouvent plusieurs de ses notes marginales, à la Préparation évangélique d'Eusèbe de Césarée, notamment pour l'établissement du texte du De Iside. Les numéros de page donnés par le traducteur convergent vers l'editio princeps procurée par Robert Estienne en 1544. Cette conclusion est confirmée par l'inventaire de la bibliothèque de l'évêque, dont le numéro 79 se lit de la sorte: «Eusebius, De preparatione et demonstratione evangelica, en grec, in-folio, impression de Robert Estienne, couvert de veau noir, prisé vingt solz ». Ici la bibliothèque du traducteur et celle de l'évêque se recoupent. Resterait à mettre la main sur cet exemplaire, dont Aulotte considérait - sur la seule foi des retouches faites dans la traduction du De placitis philosophorum en vue de la réédition de 1581 - qu'Amyot n'en avait usé qu'à partir de 1575 , tardivement donc ${ }^{38}$; la question mériterait réexamen.

De même, il est assez clair que lorsque Amyot cite en marge de ses Moralia tel locus similis de Dion Cassius, il renvoie à l'editio princeps donnée par Estienne en 1548. Même constat pour Flavius Josèphe: $1^{\prime}$ «in-folio, impression de Basle» (inventaire, $\mathrm{n}^{\circ} 77$ ) signale la princeps de la maison Froben (1544); Amyot avait en outre à sa disposition une traduction latine, « de vieille impression » $\left(\mathrm{n}^{\circ} 47\right)$.

L'enquête mériterait d'être continuée, et la future édition critique des CEuvres morales et meslées $^{39}$ nous en apprendra certainement davantage. Mais il est à peu près certain que, comme tous les érudits de son temps, Amyot ajoutait à la lecture directe des auctores celle, indirecte et de seconde main, des plus importants recueils de commentarii variés et autres 
«miscellanées» humanistes (telles celles de Budé, Érasme ${ }^{40}$, Ravisius Textor, Cælius Rhodiginus, Alessandro d'Alessandri, etc.).

\section{En marge du Banquet des sept sages} critique des Euvres morales et meslées - du Convivium septem sapientium, nous avons pu mesurer ce que l'étude des variantes marginales était en mesure de nous apprendre sur les ouvrages consultés par le traducteur. De même, Françoise Frazier, en étudiant le travail d'Amyot sur le De Pythice oraculis, avait pu confirmer certaines présomptions d'Aulotte $^{41}$, en particulier l'utilisation du Parisinus 1675 (B) - seul manuscrit disponible alors pour les traités 70-77 de Planude ${ }^{42}$ - et le recours aux variantes de Turnèbe, ainsi qu'à sa traduction enrichie de gloses philologiques ${ }^{43}$.

Le Banquet fournit un cas d'école. Les versions latines imprimées étaient nombreuses au moment où Amyot se mit au travail ; elles étaient dues, qui plus est, à des hellénistes connus de notre traducteur, directement ou indirectement. La traduction de l'Écossais Edward Henryson prit place dans le troisième tome des Moralia imprimés à Lyon par Sébastien Gryphe en 1551; l'année suivante, le Français Guillaume Plancy donnait à son tour le texte chez le même imprimeur; l'Allemand Thomas Naogeorgus (de son vrai nom Thomas Kirchmaier) publia peu après une autre traduction à Bâle, en 1556 ; enfin, Adrien Turnèbe ( $\$ 1565)$ fut à son tour le traducteur du Banquet intégré aux Moralia latins édités à Paris en 1566 - son fils Odet avait transmis le texte resté dans les papiers paternels après le décès du grand helléniste. Fortune remarquable pour ce dialogue important. Notons que la traduction de Henryson, composée entre 1549 et 1551, était dédiée à Ulrich Fugger, protecteur d'un autre Écossais, Henry Scrimger, successeur d'Amyot chez les Bochetel. Quant à Naogeorgus, c'était un autre protégé des Fugger. Pour ce qui est de Plancy et Turnèbe, ils comptaient parmi le nombre réduit des hellénistes français. Petit monde que celui des plutarquisants européens, ici réunis symboliquement autour du Banquet des sept sages - ce convivium à la faveur duquel Amyot put donner l'une de ses plus belles pages en faisant vibrer en français le mythe final d'Arion, l'un des morceaux de bravoure des Moralia.

Ce n'est pas tout ; Amyot réussit à se procurer, semble-t-il, à partir de 1566, certaines excellentes variantes du Pal. Heidel. Gr. 153 (P) grâce à Henry Scrimger, déjà mentionné ; le manuscrit en question lui aurait été transmis ou, plus vraisemblablement, quelque recueil de variantes issues de la collation de ce codex germanique ${ }^{44}$.

Mais il a échappé aux éditeurs de Plutarque comme aux spécialistes d'Amyot qu'une édition récente du texte grec du Convivium était passée, elle aussi, entre les mains et sous les yeux du traducteur. On la doit au Manceau Guillaume Plancy (Plantius) qui, après avoir traduit le Banquet en latin, en procura en 1566, dans une plaquette séparée (Paris, A. Wechel), le texte grec. 1566 : Amyot se mettait tout juste à l'ouvrage. Un grand nombre de leçons sont communes à Plancy et à Amyot - les deux éditeurs ayant tous deux pu avoir accès, à coup sûr, à l'indispensable Parisinus 1675, qui figurait en bonne place dans la bibliothèque royale de Fontainebleau. Mais le constat n'enlève rien à l'importance du relais que constitua Plancy : son édition parisienne de 1566 est à considérer comme l'un des nombreux outils de travail de notre traducteur. Et Plancy pourrait être ici, en marge du Banquet, l'un de ces anonymes désignés par la mention «alii »- mention qui pouvait

Arts et Savoirs, $10 \mid 2018$ 
aussi bien dissimuler, entre autres, tel traducteur latin cité ci-dessus, dont Amyot eût retrouvé les options philologiques par rétroversion du latin, selon une méthode déjà bien connue des humanistes.

De la bibliothèque de l'évêque à celle du traducteur, nous n'avons pu reconduire qu'imparfaitement le lecteur, en suivant parfois la piste de certaines éditions qui leur furent communes ou en rappelant l'existence d'exemplaires que l'inventaire post mortem ne put qu'ignorer. En ses livres, l'helléniste a laissé la trace insigne de son travail de longue haleine, indices ou balises pour qui désire, des siècles plus tard, lire et relire pardessus l'épaule du studieux prélat. Dans ses in-folio grecs, il nous est permis de contempler, aujourd'hui encore, le "sçavant translateur " en communication avec les humanistes de son temps - et tel qu'il ressuscitait les morts grâce aux éditions les plus fiables de leurs immortels monuments.

L'enquête ne fait que débuter en ce qui concerne cette collection assez méconnue dont l'humaniste disposa - en pointillé, imagine-t-on volontiers - de ses premières traductions à sa mort. N'étant pas en mesure d'y porter un regard véritablement panoramique, nous n'avons fait que mentionner, au risque d'y jeter une œillade myope, quelques-uns de ses exemplaires les plus éloquents, au premier rang desquels figure par exemple le volume grec des Moralia. Il est certain qu'à l'amateur d' " antiquaille ", qui désirerait comprendre pourquoi, selon les mots célèbres de Montaigne, le traducteur surpassait tous les autres auteurs par " la naïveté et la pureté de son langage », « la constance de son si long travail » - comment ne pas souligner l'expression, pesée au poids du sanctuaire - et, last but not least, « la profondeur de son savoir », il sera toujours utile de rouvrir ces insignes volumes dans lesquels la "prose d'art " d'Amyot s'est inventée, polie et ressourcée à chaque variante par le soin dévot d'un serviteur de Philologie.

\section{NOTES}

1. Sur la vie d'Amyot, voir notamment René Sturel, Jacques Amyot traducteur des "Vies parallèles " de Plutarque, Paris, H. Champion, 1909 ; Alexandre Cioranescu, Vie de Jacques Amyot, Paris, E. Droz, 1941 ; Robert Aulotte, Amyot et Plutarque. La Tradition des Moralia au XVI ${ }^{e}$ siècle, Genève, Droz, 1965 ; et le recueil Fortunes de Jacques Amyot, Michel Balard (dir.), Paris, A. G. Nizet, 1986.

2. Sylvie Le Clech-Charton, Les Vies de Jacques Amyot, édition commentée de documents inédits, Paris, Éditions du CHTS, 2013.

3. Les Vies des hommes illustres grecs et romains, Paris, chez C. Morel, 1619, f. [*** vi] r.

4. Les Vies de Jacques Amyot, op. cit., p. 143.

5. Voir François Roudaut, La bibliothèque de Pontus de Tyard: libri qui quidem extant, Paris, H. Champion, 2008.

6. Ce projet d'édition des CEuvres morales et meslées a fait l'objet d'une présentation par Olivier Guerrier: "Les Euvres morales de Plutarque », dans Anabases [revue en ligne], 3, 2006. Voir aussi id. et Françoise Frazier, "Amyot "sçavant translateur” ", dans Plutarch's Writings: Transmission, Translation, Reception, Commentary, Giovanna Pace et Paola Volpe (dir.), Napoli, M. D’Auria, 2013, p. 187-202.

7. Sur la collection des Court (ou Lecourt), voir dans ce numéro la contribution d'Hélène Lannier. 
8. Voir le catalogue Couteau-Bégarie \& Associés. Livres anciens et modernes. 27 juin $2017, \operatorname{lot}^{\circ} 40$.

9. Des œuvres de Claudien (Lyon, S. Gryphe, 1548) auraient cependant porté son chiffre et sa devise («IA SUSQUE DEQUE IA ») : voir Livres des XV \& XVI ${ }^{e}$ siècles dans leurs reliures originales, Paris, Librairie Th. Belin, 1914, $n^{\circ} 97$ : «In-16, réglé, veau fauve, dos orné, comp. de fil. dor., droits et en losange, rinceaux de feuillages et fers azurés, tr. dor.». Il est ensuite précisé : "Une reliure semblable est reproduite à la planche 47 de Libri, Monuments inédits »; voir effectivement la «vue d'artiste » reproduisant la reliure en maroquin brun d'un Suétone (Lyon, S. Gryphe, 1548) dans lesdits Monuments inédits, Londres, Dulau et $C^{\mathrm{ie}}, 1862$, pl. XLVII (reliure où, de fait, nous peinons à lire les prétendues initiales «IA»). - Nous n'avons croisé cette devise nulle part ailleurs, non plus que localisé ces volumes. Merci à notre ami Olivier Pédeflous pour ce signalement.

10. Cette « copie de Milan » [Ms. D 195 inf, f. 85r-92v] comprend des «corrections faites par Me Jacques Amyot [...] sur sa translation françoise des Vies de Plutarque marquées sur l'impression faite à Paris par Michel Vascosan en l'an MDLXV [i. e. sur la «copie de Melun » : voir infra] » et « d'autres corrections faites par ledit Amyot sur sa translation faite à Paris par ledit Vascosan en l'an MDLXXII ». Voir Robert Aulotte, Amyot et Plutarque, op. cit., p. 232 sq.

11. Voir Antoine Berman, Jacques Amyot, traducteur français. Essai sur les origines de la traduction en France, Paris, Belin, 2012.

12. Les Vies de Jacques Amyot, op. cit., p. 141-142.

13. Jean Amyot, selon le témoignage de Pierre de l'Estoile, mourut le 23 septembre 1594, «tout soudainement dans son étude, ayant sa fille auprès de lui, qui lisait dans un livre qu'il lui avait baillé ; laquelle étant sortie pour appeler, trouva à son retour son pauvre père qui avait rendu l'esprit » (cité par Robert Descimon, « La fortune de Jacques Amyot (1513-1593) : autour de Jean Amyot et de l'héritage de l'évêque d'Auxerre ", dans Fortunes de Jacques Amyot, op. cit., p. 73-86, ici p. 78-79).

14. R. Aulotte, Amyot et Plutarque, op. cit., p. 167, note 1. Voir aussi la généalogie de la famille présentée ibid., p. 364-365, ainsi que, sur l'héritage (et avec un autre arbre généalogique), Robert Descimon, art. cit.

15. Cote : 4 Y 573 INV 1006 RES. Le volume est ensuite passé dans la bibliothèque de (Florent puis de ?) Claude Chrestien qui y a apposé son ex-libris : «Cl. Christiani Q.[uinti] S.[eptimi] Flor.[entis] f.[ilii]».

16. Ces données biographiques, reportées par Amyot sur son exemplaire imprimé, attestent la consultation du Vaticanus 157 (V). Mais les variantes autographes qu'on trouve en marge de l'exemplaire ne correspondent pas aux leçons de $V$; aussi la question de l'« ancien exemplaire escript à la main » reste-t-elle entière. Voir Gerald N. Sandy, « Jacques Amyot and the manuscript tradition of Heliodorus' Aethiopica », Revue d'Histoire des Textes, nº 14-15 (1984-1985), p. 1-22 ; et Laurence Plazenet, introduction à L'Histoire cethiopique, traduction de Jacques Amyot, Paris, H. Champion, 2008, p. 22 sq.

17. Deux exceptions isolées, p. 231: un renvoi au livre xv de Strabon, sans précision; puis une

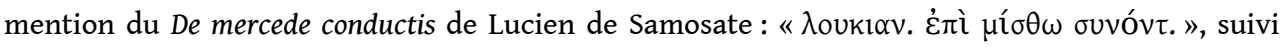
d'une mention de page: «417». Dans aucune des éditions grecques disponibles du temps d'Amyot, on ne trouve - sauf erreur - le De mercede conductis en p. 417. Amyot renvoie-t-il à un manuscrit? à une édition latine?

18. Ce fer semble être le même que celui qu'on trouve sur des Coustumes de la prevosté et vicomté de Paris (Paris, J. du Puys, 1580) possédées par Claude Dupuy : voir Jérôme Delatour, Une bibliothèque humaniste au temps des guerres de religion: les livres de Claude Dupuy, Enssib - École des chartes, 1998, $n^{\circ} 1002$, p. 232 (et frottis du médaillon en annexe, $n^{\circ} 13$, p. 270).

19. Voir R. Sturel, Jacques Amyot traducteur, op. cit., p. 135 sq. et 566 sq.

20. Melun, Bibliothèque Astrolabe, Patrimoine, Réserve $C F$, In-Folio 301 bis. On lit sur une page de garde conservée dans le dossier de restauration joint au document la mention manuscrite 
suivante : «Ce livre était à Auxerre, en 1890, chez un bouquiniste à qui je l'ai acheté. Il contient des corrections de la main d'Amyot. U. M. [Urbain Mengin]».

21. «Notes autographes d'Amyot sur un exemplaire de Justin et de Diodore » (avec trois reproductions), Bulletin du bibliophile et du bibliothécaire, 1948, p. 371-382.

22. Voir Amyot et Plutarque, op. cit. - Un tel travail avait été esquissé par l'étude pionnière de Joseph Jäger, Zur Kritik von Amyots Übersetzung der Moralia Plutarchs, Bühl, Druck der Aktiengesellschaft Konkordia, 1899.

23. Sept livres des histoires de Diodore Sicilien, Paris, M. de Vascosan, 1554, f. a iii r.

24. Voir Maria Fernanda Ferrini, « Il romanzo di Longo e la traduzione di Amyot : il problema del testo seguito ", Giornale italiano di filologia, 47, 1995, p. 77-100 ; et Laurence Plazenet, introduction à L'Histoire cethiopique, op. cit.

25. Marcel Cuvigny, «Giannotti, Turnèbe, Amyot : résultats d'une enquête sur quelques éditions annotées des Moralia de Plutarque ", Revue d'Histoire des Textes, III, 1973, p. 57-77, ici p. 75.

26. Voir R. Aulotte, Amyot et Plutarque, op. cit., p. 185-186; et Marcel Cuvigny, art. cit. - Sur cet exemplaire de Turnèbe, voir en outre Micheline Decorps-Foulquier, "À propos des différentes écritures marginales dans l'exemplaire aldin des Moralia d'Adrien Turnèbe ", Revue d'Histoire des Textes, VIII, 1978, p. 281-287.

27. Sur l'histoire et le sens de cette formule, voir Geoffrey D. Hobson, «Et Amicorum », The Library, $5^{\text {e }}$ série, 4 (1949), p. 87-99 ; et Christian Coppens, «Et amicorum : not just for friends », dans Syntagmatia. Essays on Neo-Latin Literature in Honour of Monique Mund-Dopchie and Gilbert Tournoy, Dirk Sacré et Jan Papy (dir.), Leuven University Press, 2009, p. 9-17.

28. Voir sa lettre à Lascaris de 1510, reproduite in extenso dans É. Legrand, Bibliographie hellénique, t. I, $\mathrm{n}^{\circ}$ 7, p. 332 sq., puis citée, partiellement traduite et commentée par L.-A.Sanchi, Les Commentaires de la langue grecque de Guillaume Budé, Genève, Droz, 2006, p. 126 sq. - Sur la bibliothèque de Budé, voir la contribution de Luigi-Alberto Sanchi dans ce numéro.

29. R. Sturel, Jacques Amyot, op. cit., p. 469 sq.

30. Cf. Plutarchi Choeroei de Oraculorum defectu liber, Ab Adriano Turnebo latinitate donatus, et annotationibus quibusdam illsutratus, "Ad. Turnebi in eundem librum de defectu oraculorum, Annotationes », f. 44v, lemme « Ventilabris ».

31. Histoire des Histoires, livre VI, éd. 1599, p. 359, cité par R. Sturel, Jacques Amyot, op. cit., p. 100 et

R. Aulotte, Amyot et Plutarque, op. cit., p. 182, note 7.

32. Voir Amyot et Plutarque, op. cit., p. 175 sq.

33. Cf. Variarum lectionum libri duo, Theodori Canteri Ultrajectini, Anvers, Ch. Plantin, 1574, II, 15, p. 93 sq.

34. Voir Christopher Collard, "Two Early Collectors of Euripidean Fragments: Dirk Canter and Joshua Barnes", L'Antiquité classique, 1995, 64, nº 1, p. 243-256.

35. Voir Euripide, Les Troades. Iphigénie en Aulis, traductions inédites de Jacques Amyot ; textes établis par Luigi De Nardis; préface de Jacqueline de Romilly, Naples, Bibliopolis, 1996 ; et récemment Tristan Alonge, « Les Suppliantes d'Euripide, une traduction inédite de Jacques Amyot? Rameaux, lys et dauphins ", Bibliothèque d'Humanisme et Renaissance, 2016, LXXVIII, nº 1, p. 109-126.

36. Françoise Frazier, «Amyot traducteur des CEuvres morales. Des marginalia à la version française : l'utilisation des Vies", dans Plutarque. Éditions, Traductions, Paratextes, François Frazier et Olivier Guerrier (dir.), Coimbra University Press, 2017, p. 69-86.

37. Voir la base GLN15-16 de Jean-François Gilmont, $\mathrm{n}^{\circ} 1713$.

38. Voir Amyot et Plutarque, op. cit., p. 189 et 231-232.

39. Voir la note 6 .

40. D'Érasme, l'inventaire signale, dans la bibliothèque de l'évêque, les seules paraphrases sur le Nouveau Testament $\left(\mathrm{n}^{\circ} 160\right)$ 
41. R. Aulotte, Amyot et Plutarque, op. cit., p. 172-173 : B appartenait alors à la bibliothèque de la Reine (voir Omont, Catalogue alphabétique des manuscrits de Fontainebleau, 1889, $\mathrm{n}^{\circ} 431$; et R. Sturel, Jacques Amyot, op. cit., p. 157 sq.).

42. Voir Robert Flacelière, "La tradition manuscrite des traités 70-77 de Plutarque ", Revue des Études Grecques, 65, 1952, p. 351-362 ; Mario Manfredini, «La tradizione manoscritta dei Moralia 70-77 di Plutarco », Annali della Scuola Normale Superiore di Pisa, ser. III, 6, 1976, p. 453-485; et, pour un aperçu du corpus des Moralia jusqu'à Amyot, Françoise Frazier, «Le corpus des Euvres morales, de Byzance à Amyot. Essai de synthèse », Pallas, 67, 2005, p. 77-93.

43. Voir Françoise Frazier, «Prolégomènes à une édition critique des CEuvres morales et meslées: les annotations d'Amyot au De Pythice oraculis », Exemplaria classica, 8, 2004, p. 171-193.

44. Voir R. Aulotte, Amyot et Plutarque, op. cit., p.174-175, confirmant les intuitions de Jean Defradas : voir «Une traduction de Plutarque par Amyot: le Banquet des sept sages", Revue des sciences humaines, fasc. 94, avril-juin 1959, p. 141-151; et son édition (revue par J. Hani) des Traités $n^{\circ} 10-14$ ( $n^{\circ} 13$ : Le Banquet des Sept Sages), CUF, t. II, Paris, Les Belles Lettres, 1985.

\section{RÉSUMÉS}

Pour connaître la bibliothèque de Jacques Amyot (1513-1593), nous disposons de l'inventaire qui fut réalisé après le décès de l'évêque. Cette liste, dont la plupart des exemplaires reste à identifier, n'est cependant pas suffisante pour se faire une idée exacte de la collection de livres qui fut celle du « sçavant translateur ». À cette fin, les annotations marginales portées par Amyot dans ses exemplaires de travail peuvent s'avérer extrêmement utiles. L'étude pose les bases d'une reconstitution de ce qui fut la bibliothèque de travail du grand traducteur de Plutarque.

To know the books Jacques Amyot (1513-1593) possessed, we have the inventory made after the death of the bishop. However, this list is not sufficient to accurately reconstruct the collection of the « sçavant translateur ». To this end, the marginal annotations made by Amyot in his volumes can be extremely useful. The study gives the first elements for a reconstitution of the translator's working library.

\section{INDEX}

Mots-clés : Amyot (Jacques), bibliothèque, traduction, Plutarque, hellénisme

Keywords : Amyot (Jacques), library, translation, Plutarch, hellenism

\section{AUTEUR}

ROMAIN MENINI

Université Paris-Est Marne-la-Vallée, LISAA, EA 4120 